\title{
Revealing the structure deformation in multi- component lipids by dynamic speckle pattern analysis
}

Majid Panahi

University of Zanjan

Vahideh Farzam Rad

Institute for Advanced Studies in Basic Sciences (IASBS)

Shiva Sasan

University of Zanjan

Ramin Jamali

Institute for Advanced Studies in Basic Sciences (IASBS)

Ali-Reza Moradi

Institute for Advanced Studies in Basic Sciences (IASBS)

Ahamd Darudi ( $\square$ darudi@znu.ac.ir)

University of Zanjan

\section{Research Article}

Keywords: lipid bilayers, interlayer smectic ordering, dynamic speckle pattern

Posted Date: April 9th, 2021

DOl: https://doi.org/10.21203/rs.3.rs-396278/v1

License: (c) (i) This work is licensed under a Creative Commons Attribution 4.0 International License.

Read Full License 


\title{
Revealing the structure deformation in multi-component lipids by dynamic speckle pattern analysis
}

\author{
Majid Panahi ${ }^{1,2}$, Vahideh Farzam Rad ${ }^{2}$, Shiva Sasan ${ }^{1}$, Ramin Jamali ${ }^{2}$, Ali-Reza Moradi ${ }^{2,3}$, \\ and Ahamd Darudi ${ }^{1, *}$
}

1Department of Physics, Faculty of Science, University of Zanjan, 45371-38791, Zanjan, Iran
2Department of Physics, Institute for Advanced Studies in Basic Sciences (IASBS), Zanjan 45137-66731, Iran
${ }^{3}$ School of Nano Science, Institute for Research in Fundamental Sciences (IPM), Tehran 19395-5531, Iran
"darudi@znu.ac.ir

\section{ABSTRACT}

In the bio-molecular field of Liquid-crystalline, the stacked lipid bilayers phase represents an extensive issue. It was proven that in addition to the typical smectic order, multi-component multilayer membranes could present columnar order arising from the coupling of two-dimensional intralayer phase separation. Interlayer smectic ordering distributes across hundreds of membrane lamellae, resulting in long-range alignment of phase-separated domains. In the current paper, the self-ordering of the sample over time is investigated by dynamic speckle pattern analysis. Temporal alteration of the sample is associated with changes in the intensity and contrast of the speckle pattern. We have used different factors, including motion history, co-occurrence, and time history speckle pattern, to examine the structure evolution of lipid mixtures, which are done by sequence images of speckle pattern over time. Roughness parameters inclusive of skewness and kurtosis of speckle pattern were investigated that are related to the domain size evolution of the sample. It is shown that the activity is decreasing by increasing the cholesterol content and inertia moment is inversely proportional with the growth of domain formation. The experimental setup, data recording, and data analysis are presented too.

\section{Introduction}

Multilayer lipid membranes are essential to life, perform many essential functions in biology, and regulate many cellular processes ${ }^{1}$. Multilayered lipid membranes have a variety of functional advantages over single-lipid membranes, such as to organize processes spatially, significantly increase membrane protein concentration because of an increase in surface area, and compartmentalize molecules ${ }^{2}$. Lipid bilayers composed of a ternary mixture of lipids, including sphingomyelin (SM), unsaturated phospholipid, 1,2-dioleoyl-sn-glycero-3-phos- phocholine (DOPC), and cholesterol (Chol). In bio-membranes, this kind of raft or domains for signaling activities is being used. For example, aligning the ion channels is because of ion channels localized in liquid-ordered (Lo) and aligned domains ${ }^{3}$. The alignment of domains across several bilayers may be useful in the functionality of single bilayer coupling. Phase separation into coexisting liquid-disorder (Ld) and Lo phases occur at temperatures below a miscibility transition temperature. The Ld and Lo phases are enriched in phase consisting primarily of unsaturated DOPC and enriched in Chol and SM designated, respectively. Using such bilayers, an insight into Chol-mediated phase separation has been gained ${ }^{4-7}$. The SM and Chol tend to form a Lo phase, and the accumulation of unsaturated phospholipids in a liquid-disordered phase, where the chains do not pack in a straight all-trans conformation, results in a phase transition. These roughly spherical domains appear to have important consequences in biological systems by bringing together sets of functionally interacting proteins ${ }^{8}$. The $\mathrm{Ld}$ and Lo phases transition can be seen as lipid bilayer model membranes containing a single lipid species. Membranes with a mixture of lipid species can undergo phase separation to facilitate the membrane trafficking enhancer protein insertion into the membrane. Investigation of phase separations yields information about interactions among lipid molecules ${ }^{9}$. A continuous columnar arrangement across many bilayers is formed by domains in stacked membranes aligned on top of each other. An increase in the size of domains with the number of stacked layers, proportional to the samples' thickness, results from the interlayer coupling between lamellae. Promoting domain-domain coalescence occurs over time when inter-lamellar coupling aligns vicinity domains as soon as smaller domains form independently within each bilayer when the membrane stacks are prepared ${ }^{10,11}$.

The Chol effects were investigated in a lot of studies from different points of view, such as solubility of cholesterol in lipid membranes ${ }^{12}$ and interaction of common drugs and Chol in lipid bilayers ${ }^{13}$. Chol and lipid rafts have been signified 
T-cell activation ${ }^{14,15}$ and HIV entry into T-cells by membrane fusion ${ }^{16}$. The Chol with both increasing mechanical strength and decreasing permeability plays a pivotal role in modulating the physicochemical properties of bio-membranes. Chol also can play the same position in vesicle-based delivery systems, including liposome-based drug delivery systems. However, its position in the partitioning of drug molecules to lipid membranes is poorly identified ${ }^{1}$. In general, Chol can modify the physical properties of a lipid membrane. Chol changes the mechanical property and morphology of lipid bilayers ${ }^{17}$. The presence of Chol increases the mechanical strength of the lipid bilayer ${ }^{18-21}$, which leads to increased stability and decreases inactive permeability to water, ions, and small polar molecules ${ }^{22-27}$.

Using digital holographic microscopy (DHM) for phase separation phenomena show this alignment and interlayer coupling by investigating the relative height changes. Recently, the coupling between intralayer phase separations and interlayer liquid crystal ordering in multicomponent membranes was studied, and DHM provided the information of area and volume evolution of lipid domains. It is shown that the volume and height follow the power law as the $\operatorname{area}^{3,28}$.

In biological science and medicine, several qualitative and quantitative techniques are exploited to assess the properties of the dynamic phenomenon at different scales for both basic science and clinical applications. One of the methods can be DHM to investigate the lipid bilayer examinations under different conditions of thermal and humidity situations. Besides, atomic force microscopy (AFM) is another method to measure the characteristic of different kinds of cells, which is expensive and destructive. The lipid bilayers' planar nature makes them a perfect sample for examination by AFM. Lipid phases can be detected by the AFM because of changes in bilayer thickness stemming from the differences in acyl chain packing ${ }^{29}$. Another method to examine the biological phenomenon can be considered by optical coherency tomography $(\mathrm{OCT})^{28,30-32}$. In spite of being fluorescent probes highly versatile in reporting on the membrane structure, its function possibility interacts with the membrane and becomes problematic in the measurement.

Here we are going to investigate the phase separation by dynamic speckle pattern analysis. The advantage of DHM and speckle analysis is non-invasive and non-contact. Moreover, the dynamic speckle pattern is less expensive and gives more information related to more parameters which are different from DHM. The dynamic speckle phenomenon is produced when light from a coherent source is scattered by samples with time-dependent surface activity. This activity can be due to internal or surface changes of the sample, such as particle movements, etc., causing random changes of speckle intensity. Biological samples show this behavior when they are illuminated with a laser. However, there are other dynamic processes where this behavior is also observed [46]. As stated, during phase separation, the domain surfaces are changing due to coalescence, thus giving dynamic surfaces. This activity could be due to domain motions. Immediately after the initial domain formation and coalescence, the dynamic of speckle will start. The sample preparation and experimental procedure are presented in the materials and methods section. To examine the domain formation and its coalescence in multi-component lipids, we have studied different parameters including, time history speckle pattern (THSP), co-occurrence matrix (COM), inertia moment (IM), roughness parameters, including kurtosis and skewness, and motion history image (MHI) in various concentrations that be explained in the results and discussion section. Examine these parameters for different Chol concentrations will make clear the relation between activity and different Chol concentrations.

\section{Materials and Methods}

\subsection{Sample preparation}

The samples we used for membrane multi-lamellar are nonuniform mixture systems consisting of single lipid mixtures composed of Chol, sphingomyelin (SM), unsaturated phospholipid, 1,2-dioleoyl-sn-glycero-3-phosphocholine (DOPC), as putative raft-forming ternary. The lipid multi-bilayers are prepared on the surface of clean and dry glass slide substrates. To prepare the lipid multi-bilayers, we have purchased the powder form of the lipid from Avanti ${ }^{\mathrm{TM}}$ and then dissolve the powder using chloroform. Droplets with a volume of 1 to $5 \mu 1$ of the prepared solution were put onto the cleaned glass substrate. The samples were kept in a vacuum overnight until all of the chloroform was evaporated and then were incubated for 48 to $72 h$ at $50^{\circ} \mathrm{C}$ to $60^{\circ} \mathrm{C}$ in an environment with a relative humidity of $98 \%$. In each experiment, by quick immersion of the dried plaques of multi-lamellar lipids in deionized bulk water, the phase separation regime is achieved from quenching the homogeneous phase's high temperature. In order to investigating phase separation, we varied Chol concentration in equimolar mixtures of SM and DOPC systematically (1:1 SM/DOPC $+\mathrm{xChol} ; \mathrm{x}=10-30 \%$ (molar)). These lipid mixtures are known to phase-separate into two coexisting liquid phases when organized as single bilayers under their miscibility transition temperature.

\subsection{Experimental procedure}

Figure 1(a) shows the schematic of the experimental procedure for recording dynamic speckle patterns. The laser beam (He-Ne Laser, $632.8 \mathrm{~nm}, 5 \mathrm{~mW}$ ) is expanded by the beam expander BE, then the speckle pattern produced by diffuser D (Thorlabs, DG05-120-MD) is reflected by mirror $\mathrm{M}$ on the sample through the condenser (Thorlabs, CSC2001, Achromatic, NA=0.78, $\mathrm{WD}=6.6 \mathrm{~mm}$ ). The sample is immediately moved to the microscopy stage after immersion in humidity and temperature for 48-72 hours. The domains are formed and evolved in time. The generated speckle patterns are pass through the microscope 

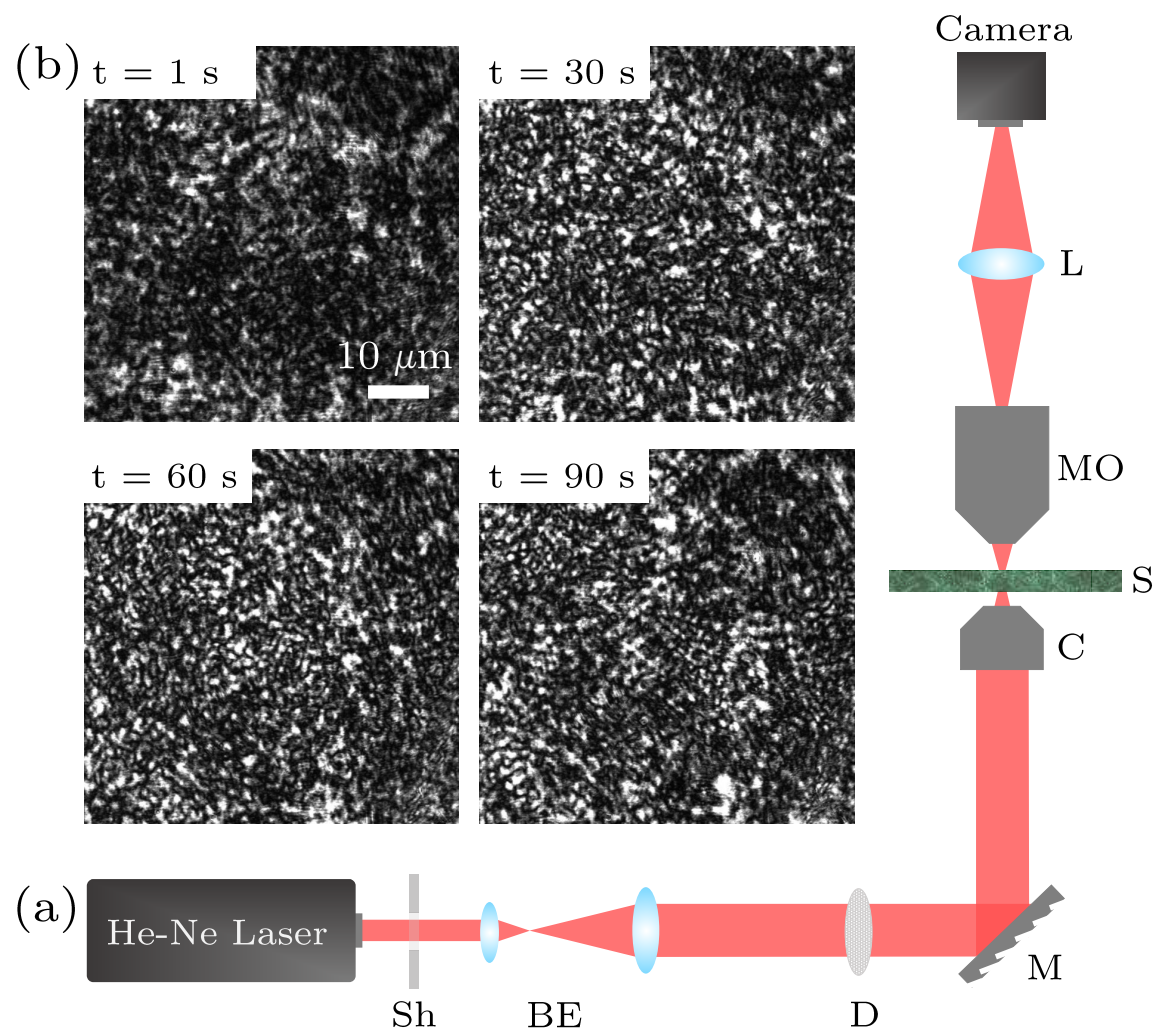

(a)
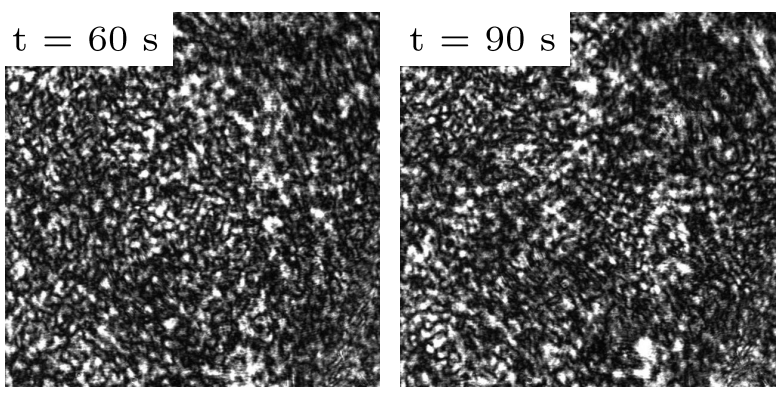

Figure 1. (a) Experimental setup schematically for dynamic speckle capture; Sh: shutter, BE: beam expander, D: Diffuser, M: mirror, C: Condenser, S: Sample, MO: Microscope Objective, L: collecting lens. (b) Speckle patterns of domain evolution over times.

objective MO (Olympus, 40X, Plan Achromat, NA=0.65, WD=0.6 mm) and recorded by a digital camera (DCC1545M, Thorlabs, 8-bit dynamic range, $5.2 \mu \mathrm{m}$ pixel pitch) with a collecting lens (L). $\mathrm{L}$ has a focal length of $50 \mathrm{~mm}$. The digital camera is set to record $1280 \times 1024$ pixels under its central region with an exposure time of $0.22 \mathrm{~ms}$. The speckle patterns are recorded in video format for 90 seconds with a $25 \mathrm{fps}$ frame rate. It should be noted that the $\mathrm{He}-\mathrm{Ne}$ laser possesses adequate coherency and stability. The laser is turned on at least half an hour before the operation to ensure intensity stability, which is highly significant in speckle analysis. Using the shutter (Sh), the exposure time of the sample is controlled.

In order to ensure the low effect of noise, such as vibration, time-dependent contaminants in optical elements, and background environmental light, a controlling experiment is conducted. For control data, the experimental conditions such as temperature are kept constant and the specimen is fixed. Since only a part of the specimen area is owned by the lipid specimen, an empty area in the vicinity of the specimen, is taken as a reference area. The speckles analysis procedures that are presented in this paper is applied to the reference data. The results of the controlling experiment are presented in Supplementary Material.

\subsection{Numerical processing}

An encouraging optical method for evaluating biological processes is dynamic laser speckle. A beneficial description of the surface properties of the sample is provided through dynamic speckle analysis in the characterization procedure field. Different analysis results could reveal the sample's activity, particularly in biomaterials, which facilize quantitative and qualitative information on the processes making dynamic changes in the sample under examination. The objectivity of the present research is to characterize the phase separation activity of ternary lipids mixture as a function of time. The recorded speckle patterns are numerically processed, and multiple useful statistical parameters defined in this section are calculated ${ }^{33,34}$. Illuminating an active object by a laser causes dynamics speckle patterns. The static speckle pattern is possible when the wavelength and intensity of the laser are stable, and also sample has no movement. Therefore, when the wavelength and the intensity of the laser are stable, mobility detected by the dynamic laser speckle in biomaterials is due to their internal feature, for example, growth and cell division, biochemical reactions, water-related activities, as well as scaffold activity detection ${ }^{35-41}$. In phase separation of domains, the activity-related measurements, which can be in terms of coalescing of domains, are associated with the interlayer alignment of intralayer domains' structure of multi-component lipids ${ }^{3,28}$. We name the mentioned structural 
variations as "domain-domain coalescence activities". The time history speckle pattern (THSP) is a two-dimensional matrix that representing the time evolution of a set of $M$ points, so-called image datapack points, in sequential speckle patterns, where $M$ represents the number of points in the THSP matrix, which is equal to the raws number of the image in the datapack. Also, the THSP has $N$ columns, where $N$ represents the number of images in the datapack. Thereby, a raw of the THSP matrix show this set of points in a speckle pattern, while its columns show the time evolution. From the initial pattern, the set of $M$ points is randomly chosen to reconstruct the first column of the THSP matrix. The corresponding points in successive patterns are used for constructing the other columns of the THSP matrix.

The THSP provides a graphical sign of the sample activity level that the higher the THSP line variation represents the higher activity of the sample under study ${ }^{42}$. The concept of the THSP is the foundation of numerical results exemplary co-occurrence matrix (COM), inertia moment (IM), and so on. In addition, several parameters can be investigated independently of using the THSP, such as motion history image (MHI), roughness parameters (kurtosis, skewness, etc.), contrast, and homogeneity related to activity types ${ }^{43}$.

$\mathrm{COM}$ is an intermediary matrix, which evaluated the dispersion of successive pixels in a THSP of $M$ points monitoring by $N$ speckle patterns. COM demonstrates a transition intensities histogram:

$$
\operatorname{COM}(i, j)=\sum_{m=1}^{M} \sum_{n=1}^{N-1} \begin{cases}1, & \text { if } \operatorname{THSP}(m, n)=i \\ \text { and } \operatorname{THSP}(m, n+1)=j & \text { otherwise. }\end{cases}
$$

IM is considered as statistical and numerical activity indicator that is calculated by Eq. 1:

$$
\mathrm{IM}=\sum_{i} \sum_{j} \frac{\operatorname{COM}(i, j)}{\sum_{m} \operatorname{COM}(i, m)}|i-j|^{2} .
$$

In order to reduction of the inhomogeneities effect in the analyzed images, the normalization is applied in a way that the sum of values in each line of the COM equal to 1 . The inertia moment's name is derived from the mechanical resembling of this operation.

The average alternation of intensities to mean value of data is defined as $R_{P 1}$, while definition the standard deviation of the distribution is the root mean square of intensities $\left(R_{P 2}\right)$ :

$$
\begin{aligned}
& R_{P 1}=\frac{1}{P Q} \sum_{p=1}^{P} \sum_{q=1}^{Q}|I(p, q)-\langle I(p, q)\rangle|, \\
& R_{P 2}=\left[\frac{1}{P Q} \sum_{p=1}^{P} \sum_{q=1}^{Q}[I(p, q)-\langle I(p, q)\rangle]^{2}\right]^{\frac{1}{2}},
\end{aligned}
$$

where $P$ and $Q$ are the horizontal and vertical sizes of the speckle patterns, $p$ and $q$ count the pixel numbers, and $I$ is the intensity all over the speckle patterns $R_{P 1}$. The $R_{P 1}$ is called the average roughness. These metrics can be used to providing a general distribution roughness estimation. Equivalent, skewness, $R_{P 3}$, and kurtosis, $R_{P 4}$, are other usual roughness parameters that can be used to evaluate the samples:

$$
\begin{aligned}
& R_{P 3}=\frac{1}{P Q R_{P 2}^{3}} \sum_{p=1}^{P} \sum_{q=1}^{Q}[I(p, q)-\langle I(p, q)\rangle]^{3}, \\
& R_{P 4}=\frac{1}{P Q R_{P 2}^{4}} \sum_{p=1}^{P} \sum_{q=1}^{Q}[I(p, q)-\langle I(p, q)\rangle]^{4} .
\end{aligned}
$$

According to the definition of $R_{P 3}$, which is the third moment of the deviation from the mean value, it is a measure of the symmetry's degree related to the intensities distribution. Positive skewness represents a "peaky" distribution, while negative skewness represents a superiority of valleys, i.e., low intensities. On the other hand, $R_{P 3}=0$ represents symmetric intensity distribution of surface. The parameter that measures the distribution sharpness all over the pattern is kurtosis, $R_{P 4}$. Kurtosis is the indicator of the intensity distribution width ${ }^{44}$. Another parameter that is related to the activity of the samples is the Motion History Image (MHI). It is used to characterize movements within a row of images in time. The MHI indicates a static pattern that the pixel intensity is a function of the recent image sequence motion and contains the fundamental information in order to determine the motion of the object during the activity ${ }^{45,46}$. MHI has been used profoundly for visualizing the perfusion changes locations during different stimuli ${ }^{47}$. Furthermore, MHI finds out the object's motion depends on the pixel's timestamps 
(a)

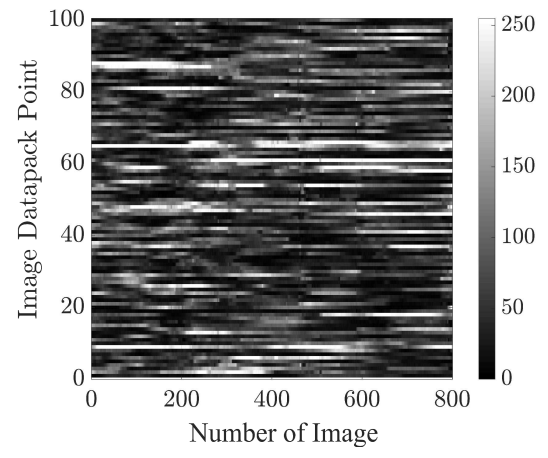

(b)

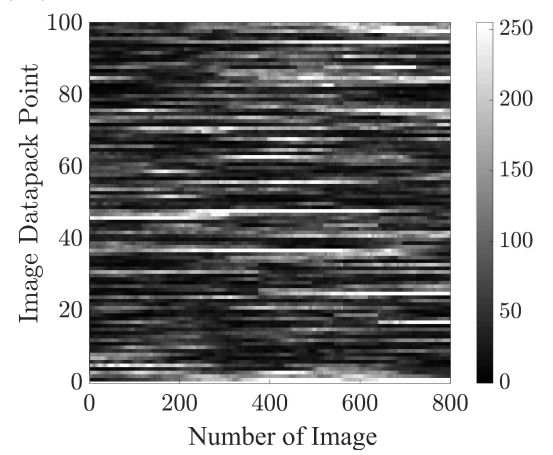

(c)



Figure 2. Time history speckle pattern (THSP) for (a) 10\%, (b) 20\%, and (c) 30\% Chol. THSP formed by tracking 100 random points throughout a collection of 810 speckle patterns of lipid (image datapack points). Speckle pattern recorded every second while domain evolution.

(a)

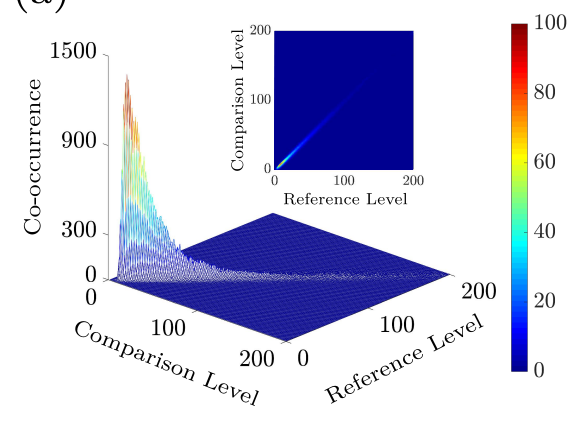

(b)



(c)

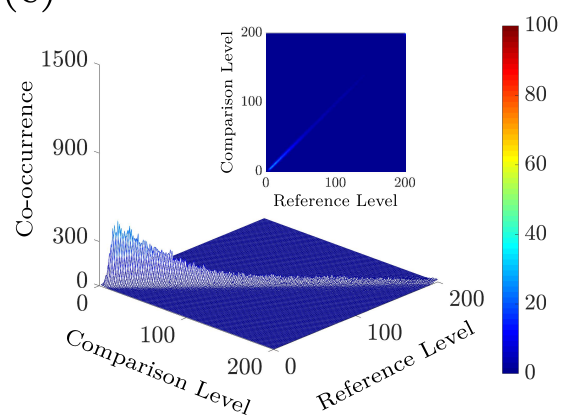

Figure 3. 3D plot and 2D map of co-occurrence matrix (COM) of the associated THSPs in Fig. 2 for (a) 10\%, (b) 20\%, and (c) $30 \%$ Chol.

through a set of images according to a motion sequence. For analyzing the MHI as input data, datapack is used, which is a 3D matrix created by grouping intensity matrices. The sequential object images showing changes are compared using the following subtraction:

$$
S_{k}=I_{k}-I_{k-1},
$$

where $I_{k}$ and $I_{k-1}$ are the grayscale images in the moments $k$ and $k-1$. Meanwhile, the resulting image $\left(S_{k}\right)$ is the silhouette of two consecutive images. A threshold must be applied for each pixel $(m, n)$ as follows:

$$
T_{k}(m, n)= \begin{cases}1 & \text { if }\left|S_{k}(m, n)\right|>U \\ 0 & \text { if }\left|S_{k}(m, n)\right| \leqslant U,\end{cases}
$$

where $T_{k}(m, n)$ is the threshold image of $S_{k}$ at each moment $k$, and $U$ is the activity threshold parameter. Therefore, MHI considers the pixel activity that has an intensity larger than $U$. Finally, the motion history of the object is therefore recorded by the MHI procedure, in the instant $k$, through Eq. (9):

$$
M H I=255 \sum_{l=0}^{N} T_{k-l} h_{l},
$$

the value of $h_{l}$ is equal to:

$$
h_{l}=\frac{N-l}{\mathrm{M}},
$$

where the $h_{l}$ value is the weighting parameter that is based on the image's age, and $\mathrm{M}=N(N+1) / 2$. 
(a)



(b)

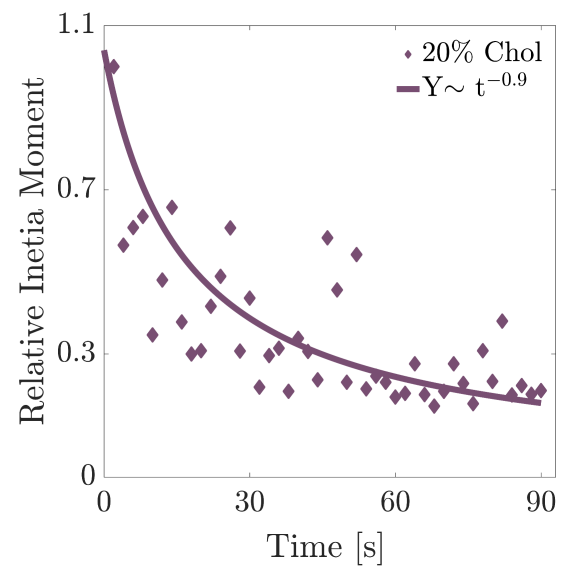

(c)

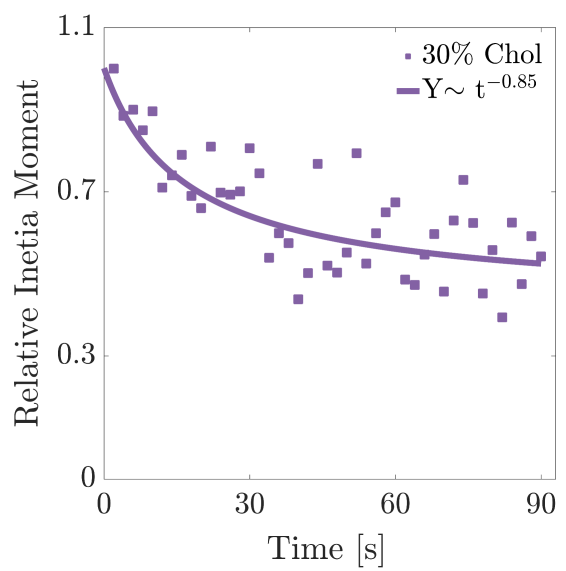

Figure 4. Inertia moment (IM) of lipid samples' THSP as a function of time for (a) $10 \%$, (b) $20 \%$, (c) $30 \%$ Chol. The power-law function is fitted on each graph.

\section{Results and discussion}

After recording the speckle pattern of domain evolution, we have analyzed the speckle image sequence derived from the recorded movies. As it is shown in Fig. 1(b), while the domains are coalescing, the speckle patterns change. This evolution is because of phase separation in the stacked lipid bilayer. Figures 2(a), 2(b), and 2(c) show the THSP matrices for samples with different Chol content (concentrations of Chol combined with the lipid sample). THSPs are built by putting together the intensity of 100 random pixels throughout a collection of 810 speckle patterns. The fluctuations in the intensity of the pixels will be the result of the activity of lipids related to coalescing. As the increasing percentage of Chol concentration, the amplitude of the intensity of horizontal lines decreases, which indicates the relatively lower activity of the lipid by adding Chol concentration of the solution. In 30\% and higher Chol concentration, the THSP pattern approaches a structure with relatively uniform intensity and shows less activity.

Moreover, for better understanding, the change in the lipids' activity, the associated COM matrices of the samples are calculated at the aforementioned Chol concentration. Figures 3(a), 3(b), and 3(c) show the 3D plot and 2D map of COM matrix of the THSPs of lipid's domain evolution (indicated in Fig. 2), at 10\%,20\%, and 30\% Chol, respectively. The original images are 8-bit, and due to lack of data, the axes are limited to 200. Figure 3 shows that by increasing the Chol, the width of the pattern through the diagonal elements is increased and also shows a lower peak value is an indication of lower activities during domain coalescing. Accordingly, the distributions around the principal diagonal, non-zero elements of the COM matrices represent the evolution of the sample with different Chol concentrations. To provide a quantitative assessment of the spread of the COM values around the principal diagonal as an activity indicator for the lipid samples, the IM values of several samples are computed as a function of time. Figures 4(a), 4(b), and 4(c) show the IM value of the lipids during $90 \mathrm{~s}$ for 10\%, 20\%, and $30 \%$ Chol, respectively. It should be noted that in Fig. 4, each point is the average value of IM for two seconds intervals. IM is defined as summation on the squared row distance to the principal diagonal of THSP; therefore, it is the right quantitative illustration to the "cloudiness", such as the amount of data spreading of COM distribution around its principal diagonal. The larger width is proportional to higher activity. Figure 4 shows the decreasing IM values and activity of the lipid samples in various concentrations at different times. The decrease of the IM is an indication of lower activity that can be attributed to the less mobility due to big domain size resulting in domain-domain coalescence. It is clear in Fig. 4, there is a general downward change as a function of time. Moreover, It has shown that the average area of domains is increasing over time ${ }^{3,28}$, which makes the surface more uniform and causes a decrease in the roughness of the sample or IM value. Therefore, we believe that the change of IM is proportional to the inverse of the growth of domain formation. In order to prove it, a power-law function is fitted on IM graphs in Fig. 4. The exponent is about -0.9 , in which the positive exponent is in agreement with the reported value $^{3}$. According to reports, the temporal evolution of the average area of domains in bilayers follows the universal growth law, $h \sim t^{2 n}$, and the growth exponent, $n$, is $1 / 3<n<1 / 2^{3,28}$.

The lipid activity and coalescence are associated with the surface structure that is given by surface roughness. Here we examined the roughness parameters by calculating the kurtosis and skewness for the samples. Kurtosis and skewness values are generally interpreted together. For a sample with $20 \% \mathrm{Chol}$, the kurtosis parameter for $1 \mathrm{~s}, 30 \mathrm{~s}, 60 \mathrm{~s}$, and $90 \mathrm{~s}$ time intervals are shown in Figs. 5(a), 5(b), 5(c), and 5(d), respectively. Also, the skewness parameter for 1s, 30s, 60s, and 90s time intervals are 

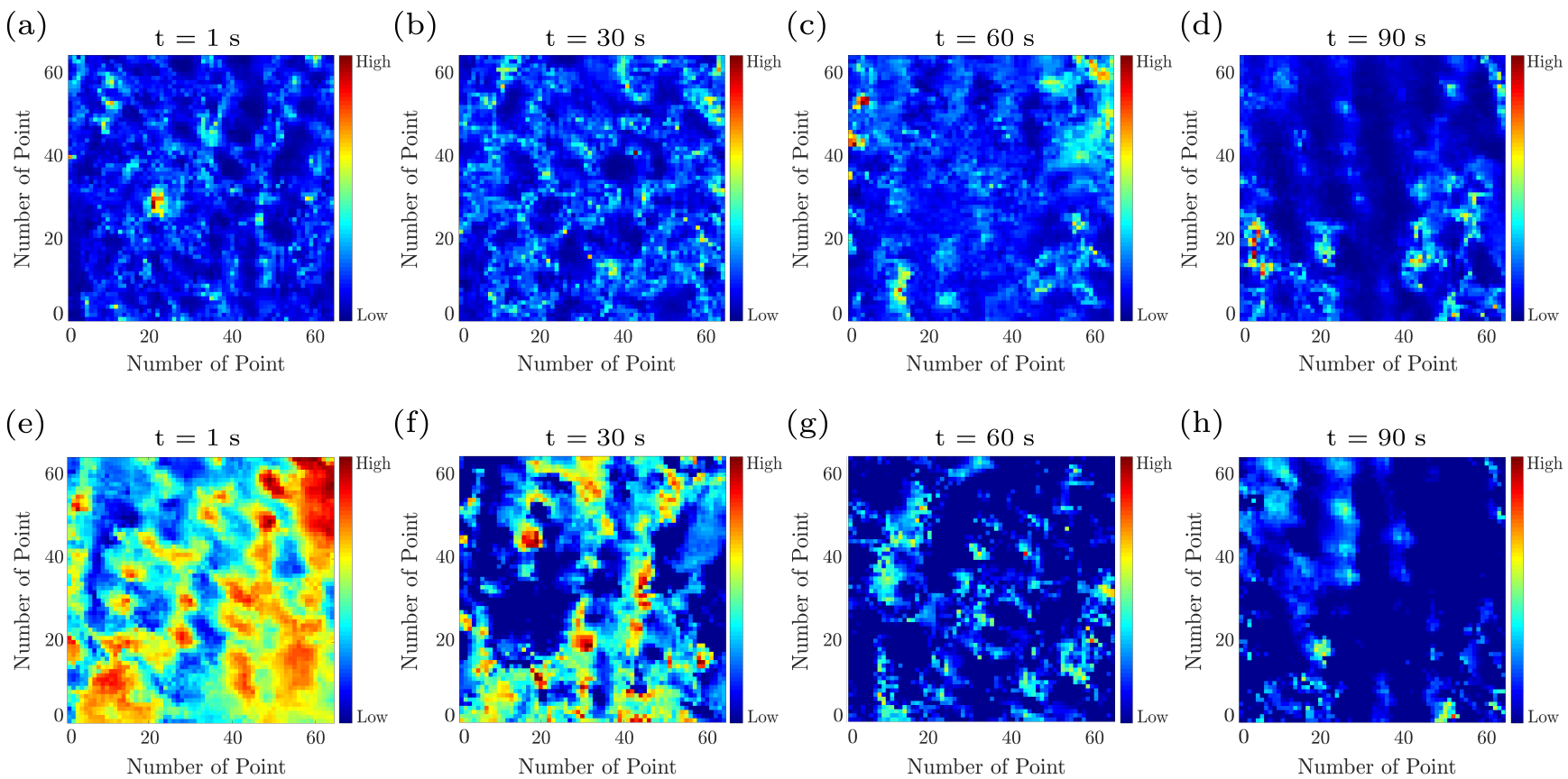

Figure 5. (a), (b), (c), and (d) Temporal speckle kurtosis matrix of 20\% Chol. (e), (f), (g), and (h) Temporal speckle skewness matrix of $20 \%$ Chol.

(a)



(b)

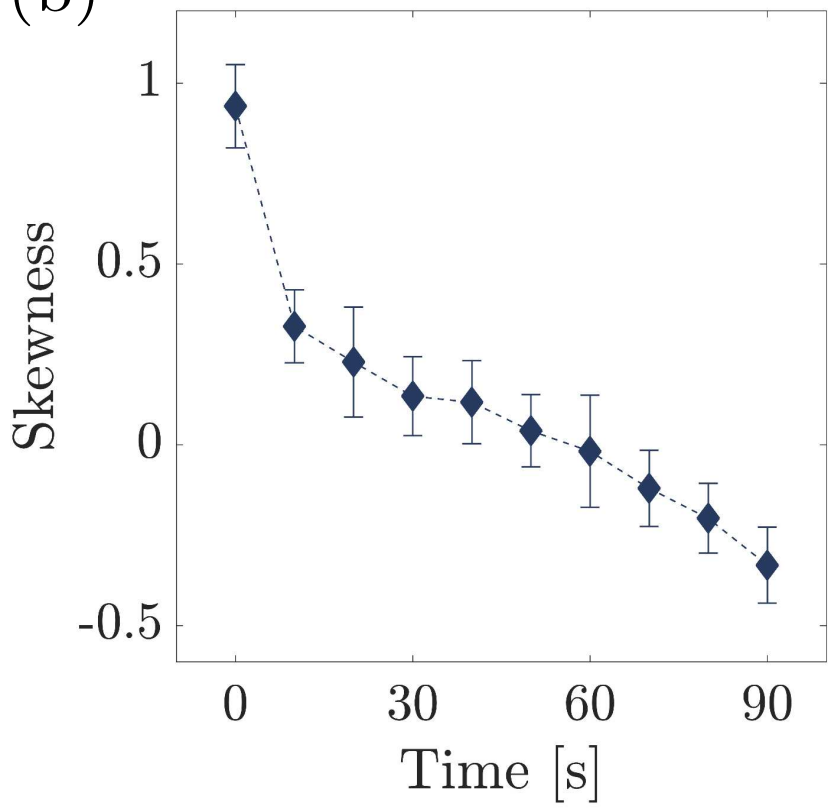

Figure 6. (a) Average kurtosis, and (b) Average skewness of 100 speckle patterns of lipid surface as a function of time at $20 \%$ Chol.

shown in Figs. 5(e), 5(f), 5(g), and 5(h), respectively.

In order to reduce the computational time, a portion of the original speckle pattern with 64 by 64 pixels is selected for calculations. The blue pixels in which indicating less activity, are increased over time. It is obvious that the changes in skewness are greater than kurtosis.

For better demonstration, the average value of the kurtosis and skewness are shown in Figs. 6(a) and 6(b), respectively. 
(a)

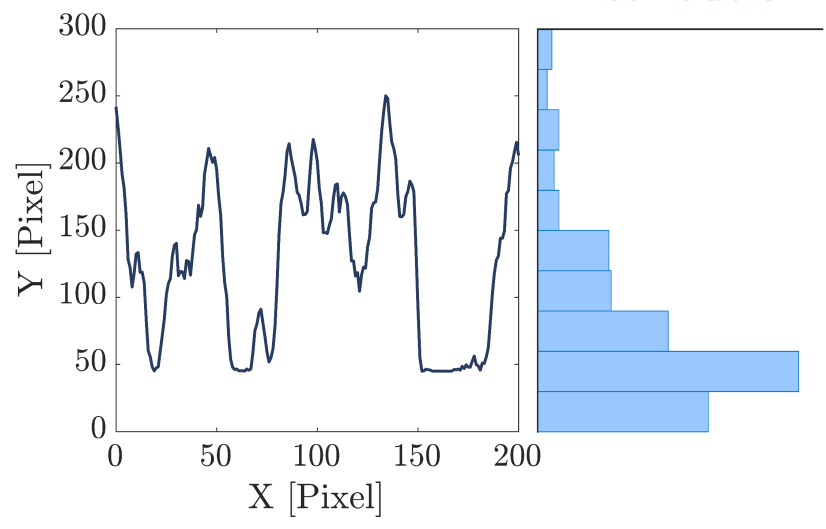

(b)

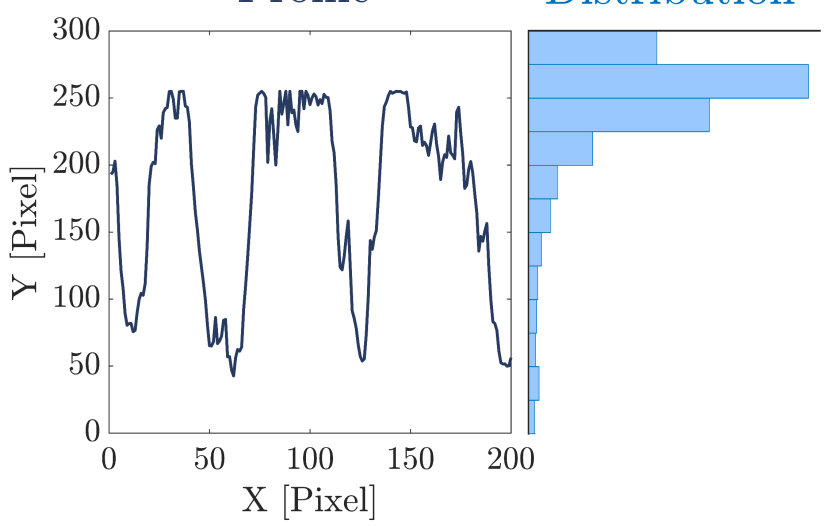

Figure 7. The intensity profile of two speckle patterns and corresponding intensity histograms at two different times, (a) $t=10 \mathrm{~s}$ and (b) $t=90$ s, illustrate positive and negative skewness respectively.

(a)

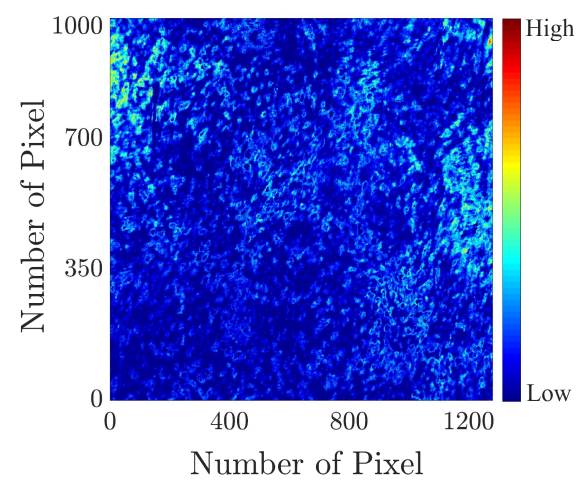

(b)

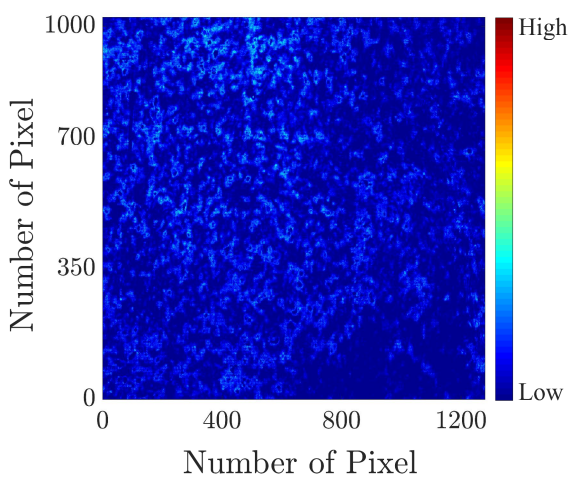

(c)

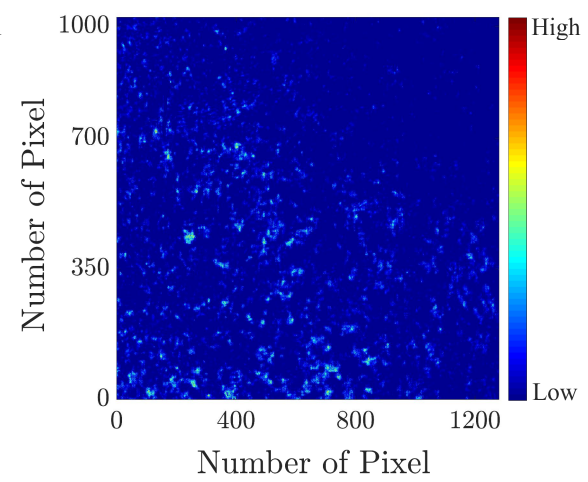

Figure 8. Motion history image (MHI) for (a) 10\%, (b) 20\%, (c) 30\% Chol. Each MHI was generated from a stack of speckle pattern images.

The same behaviour for kurtosis and skewness for different Chol concentrations were observed. Each error bar is obtained by calculating the kurtosis and skewness values over 100 speckle patterns. The variation of kurtosis and skewness shows that the surface of the samples' gets smoother in longer times due to the domain coalescence and enlarging the domains.

Figure 7 shows the profile of the speckle pattern at the first and final time and corresponding intensity distributions. In ${ }^{48,49}$, it is illustrated that in a dynamic surface, by increasing the number of bright pixels, the roughness of the surface is decreasing. The profiles of the speckle patterns in Fig. 7 clearly show that at the beginning the number of bright pixels are less and then becomes larger at last. Figure 7(a) and 7(b) show the lower bright pixels (positive skewness) and the higher bright pixels (negative skewness), respectively. For quantitative purposes, it seems the same interpretation can be presented by calculating the skewness. The behaviour of skewness of the speckle pattern in Fig. 6(b), shows that at the beginning it is positive then becomes negative at last. Therefore, we believe, the skewness of the speckle pattern is a good parameter to show the evolution of the surface roughness.

Figure 8 shows the MHI images generated from a stack of speckle patterns. During increasing the concentration of Chol, there is an effect that could be detected by following the MHI maps. MHI is a method used to recognize motion within a row of images through time. In the MHI maps, the background was illustrated in blue, and the higher density of dots demonstrated a great change in the activity, while the smaller density of dots presented low activity. According to Fig. 8, the motion of domains or activity decreased by increasing the concentration of Chol, as well as shows the movement of the domain while coalescing. Therefore, by following the MHI images, it becomes possible to demonstrate the surface activity and domain evolutions over time.

In Supplementary Material, Figs. S1-S3, we have also presented a similar investigation of THSP, COM, MHI, IM, and 
roughness parameters for controlling the experiment.

\section{Conclusion}

The dynamic speckle analysis has been used for measuring the self-ordering epitaxial films of stacked lipid bilayers. Different factors such as time history, co-occurrence, inertia moment, roughness parameters, and motion history image of speckle patterns have been used to show the rate of activity over time and also at different the Chol concentration. It is observed that less activity happens at higher concentrations in which predict the liquid-liquid coexistence is abandoned at higher Chol concentrations $(\mathrm{x}>$ $40 \%$ ). According to the results, we suggest that the change of inertia moment can be proportional to the inverse of the growth of domain formation and the trend of these changes is related to the temporal evolution of the average area of domains. Also, we believe that the skewness of the speckle pattern is a good parameter to show the roughness evolution of the surface.

\section{Disclosures}

The authors declare no conflicts of interest.

\section{Acknowledgements}

The authors would like to thank Dr. Lobat Tayebi for the preparation of the samples.

\section{References}

1. Poojari, C. et al. Cholesterol reduces partitioning of antifungal drug itraconazole into lipid bilayers. The J. Phys. Chem. B 124, 2139-2148 (2020).

2. Heath, G. R. et al. Multilayered lipid membrane stacks for biocatalysis using membrane enzymes. Adv. Funct. Mater. 27, 1606265 (2017).

3. Tayebi, L. et al. Long-range interlayer alignment of intralayer domains in stacked lipid bilayers. Nat. materials 11, 1074-1080 (2012).

4. Veatch, S. L. \& Keller, S. L. Organization in lipid membranes containing cholesterol. Phys. review letters 89, 268101 (2002).

5. McConnell, H. M. \& Vrljic, M. Liquid-liquid immiscibility in membranes. Annu. review biophysics biomolecular structure 32, 469-492 (2003).

6. Hammond, A. et al. Crosslinking a lipid raft component triggers liquid ordered-liquid disordered phase separation in model plasma membranes. Proc. Natl. Acad. Sci. 102, 6320-6325 (2005).

7. Bacia, K., Schwille, P. \& Kurzchalia, T. Sterol structure determines the separation of phases and the curvature of the liquid-ordered phase in model membranes. Proc. Natl. Acad. Sci. 102, 3272-3277 (2005).

8. Brown, D. \& London, E. Functions of lipid rafts in biological membranes. Annu. review cell developmental biology 14, 111-136 (1998).

9. Elson, E. L., Fried, E., Dolbow, J. E. \& Genin, G. M. Phase separation in biological membranes: integration of theory and experiment. Annu. review biophysics 39, 207-226 (2010).

10. Bechinger, B. Domains stack up. Nat. Mater. 11, 1005-1006 (2012).

11. Ursell, T. S., Klug, W. S. \& Phillips, R. Morphology and interaction between lipid domains. Proc. Natl. Acad. Sci. 106, 13301-13306 (2009).

12. Barrett, M. A. et al. Solubility of cholesterol in lipid membranes and the formation of immiscible cholesterol plaques at high cholesterol concentrations. Soft Matter 9, 9342-9351 (2013).

13. Alsop, R. J. et al. Cholesterol expels ibuprofen from the hydrophobic membrane core and stabilizes lamellar phases in lipid membranes containing ibuprofen. Soft matter 11, 4756-4767 (2015).

14. Gaus, K., Chklovskaia, E., Fazekas de St. Groth, B., Jessup, W. \& Harder, T. Condensation of the plasma membrane at the site of t lymphocyte activation. The J. cell biology 171, 121-131 (2005).

15. Biancotto, A. et al. Hiv-1-induced activation of cd4+ $\mathrm{t}$ cells creates new targets for hiv-1 infection in human lymphoid tissue ex vivo. Blood, The J. Am. Soc. Hematol. 111, 699-704 (2008). 
16. Campbell, S., Crowe, S. M. \& Mak, J. Lipid rafts and hiv-1: from viral entry to assembly of progeny virions. J. Clin. Virol. 22, 217-227 (2001).

17. Gao, Q., Wu, G. \& Lai, K. W. C. Cholesterol modulates the formation of the a $\beta$ ion channel in lipid bilayers. Biochemistry 59, 992-998 (2020).

18. Pan, J., Tristram-Nagle, S. \& Nagle, J. F. Effect of cholesterol on structural and mechanical properties of membranes depends on lipid chain saturation. Phys. Rev. E 80, 021931 (2009).

19. Khatibzadeh, N., Gupta, S., Farrell, B., Brownell, W. E. \& Anvari, B. Effects of cholesterol on nano-mechanical properties of the living cell plasma membrane. Soft Matter 8, 8350-8360 (2012).

20. Khelashvili, G. \& Harries, D. How cholesterol tilt modulates the mechanical properties of saturated and unsaturated lipid membranes. The J. Phys. Chem. B 117, 2411-2421 (2013).

21. Al-Rekabi, Z. \& Contera, S. Multifrequency afm reveals lipid membrane mechanical properties and the effect of cholesterol in modulating viscoelasticity. Proc. Natl. Acad. Sci. 115, 2658-2663 (2018).

22. Saito, H. \& Shinoda, W. Cholesterol effect on water permeability through dppc and psm lipid bilayers: a molecular dynamics study. The J. Phys. Chem. B 115, 15241-15250 (2011).

23. Milianta, P. J., Muzzio, M., Denver, J., Cawley, G. \& Lee, S. Water permeability across symmetric and asymmetric droplet interface bilayers: interaction of cholesterol sulfate with dphpc. Langmuir 31, 12187-12196 (2015).

24. Issack, B. B. \& Peslherbe, G. H. Effects of cholesterol on the thermodynamics and kinetics of passive transport of water through lipid membranes. The J. Phys. Chem. B 119, 9391-9400 (2015).

25. Shinoda, W. Permeability across lipid membranes. Biochimica et Biophys. Acta (BBA)-Biomembranes 1858, 2254-2265 (2016).

26. Dotson, R. J., Smith, C. R., Bueche, K., Angles, G. \& Pias, S. C. Influence of cholesterol on the oxygen permeability of membranes: insight from atomistic simulations. Biophys. journal 112, 2336-2347 (2017).

27. Lopez, M. et al. Effects of acyl chain unsaturation on activation energy of water permeability across droplet bilayers of homologous monoglycerides: Role of cholesterol. Langmuir 34, 2147-2157 (2018).

28. Farzamrad, V., Moradi, A.-R., Darudi, A. \& Tayebi, L. Digital holographic microscopy of phase separation in multicomponent lipid membranes. J. biomedical optics 21, 126016 (2016).

29. Connell, S. D. \& Smith, D. A. The atomic force microscope as a tool for studying phase separation in lipid membranes. Mol. membrane biology 23, 17-28 (2006).

30. Rebelo, L. M., de Sousa, J. S., Mendes Filho, J. \& Radmacher, M. Comparison of the viscoelastic properties of cells from different kidney cancer phenotypes measured with atomic force microscopy. Nanotechnology 24, 055102 (2013).

31. Engler, A. J., Rehfeldt, F., Sen, S. \& Discher, D. E. Microtissue elasticity: measurements by atomic force microscopy and its influence on cell differentiation. Methods cell biology 83, 521-545 (2007).

32. Schmitt, J. M. Optical coherence tomography (oct): a review. IEEE J. selected topics quantum electronics 5, 1205-1215 (1999).

33. Braga, R. A., González-Peña, R. J., Viana, D. C. \& Rivera, F. P. Dynamic laser speckle analyzed considering inhomogeneities in the biological sample. J. Biomed. Opt. 22(4), 045010 (2017).

34. Murialdo, S. et al. Analysis of bacterial chemotactic response using dynamic laser speckle. J. Biomed. Opt. 14, 064015 (2009).

35. Braga, R. A., Dupuy, L., Pasqual, M. \& Cardoso, R. Live biospeckle laser imaging of root tissues. Eur. Biophys. J. 38(5), 679-686 (2009).

36. Cardoso, R. \& Braga, R. Enhancement of the robustness on dynamic speckle laser numerical analysis. Opt. Lasers Eng. 63, 19-24 (2014).

37. Grassi, H. C. et al. Quantitative laser biospeckle method for the evaluation of the activity of trypanosoma cruzi using vdrl plates and digital analysis. PLoS Neglected Trop. Dis. 10(12), e0005169 (2016).

38. Ramírez-Miquet, E. E. et al. Digital imaging information technology for biospeckle activity assessment relative to bacteria and parasites. Lasers Med. Sci. 32(6), 1375-1386 (2017).

39. Zdunek, A., Adamiak, A., Pieczywek, P. M. \& Kurenda, A. The biospeckle method for the investigation of agricultural crops: A review. Opt. Lasers Eng. 52, 276-285 (2014). 
40. Vladimirov, A. et al. Applicaton of speckle dynamics for studies of cell metabolism. In Optical Methods for Inspection, Characterization, and Imaging of Biomaterials II, vol. 9529, 95291F (International Society for Optics and Photonics, 2015).

41. Rad, V. F., Panahi, M., Jamali, R., Darudi, A. \& Moradi, A.-R. Non-invasive in situ monitoring of bone scaffold activity by speckle pattern analysis. Biomed. optics express 11, 6324-6336 (2020).

42. Braga, R., Silva, W., Sáfadi, T. \& Nobre, C. Time history speckle pattern under statistical view. Opt. Commun. 281(9), 2443-2448 (2008).

43. Dhandayuthapani, B., Yoshida, Y., Maekawa, T. \& Kumar, D. S. Polymeric scaffolds in tissue engineering application: a review. Int. J. Polym. Sci. 2011 (2011).

44. Gadelmawla, E., Koura, M., Maksoud, T., Elewa, I. \& Soliman, H. Roughness parameters. J. Mater. Process. Technol. 123(1), 133-145 (2002).

45. Davis, J. W. Hierarchical motion history images for recognizing human motion. In Proceedings IEEE Workshop on Detection and Recognition of Events in Video, 39-46 (IEEE, 2001).

46. Ansari, M. Z., Ramírez-Miquet, E. E., Otero, I., Rodríguez, D. \& Darias, J. G. Real time and online dynamic speckle assessment of growing bacteria using the method of motion history image. J. Biomed. Opt. 21, 066006 (2016).

47. Ansari, M. Z., Humeau-Heurtier, A., Offenhauser, N., Dreier, J. P. \& Nirala, A. K. Visualization of perfusion changes with laser speckle contrast imaging using the method of motion history image. Microvasc. research 107, 106-109 (2016).

48. Kayahan, E., Oktem, H., Hacizade, F., Nasibov, H. \& Gundogdu, O. Measurement of surface roughness of metals using binary speckle image analysis. Tribol. Int. 43, 307-311 (2010).

49. Fuh, Y.-K., Hsu, K. C. \& Fan, J. R. Roughness measurement of metals using a modified binary speckle image and adaptive optics. Opt. Lasers Eng. 50, 312-316 (2012). 


\section{Figures}
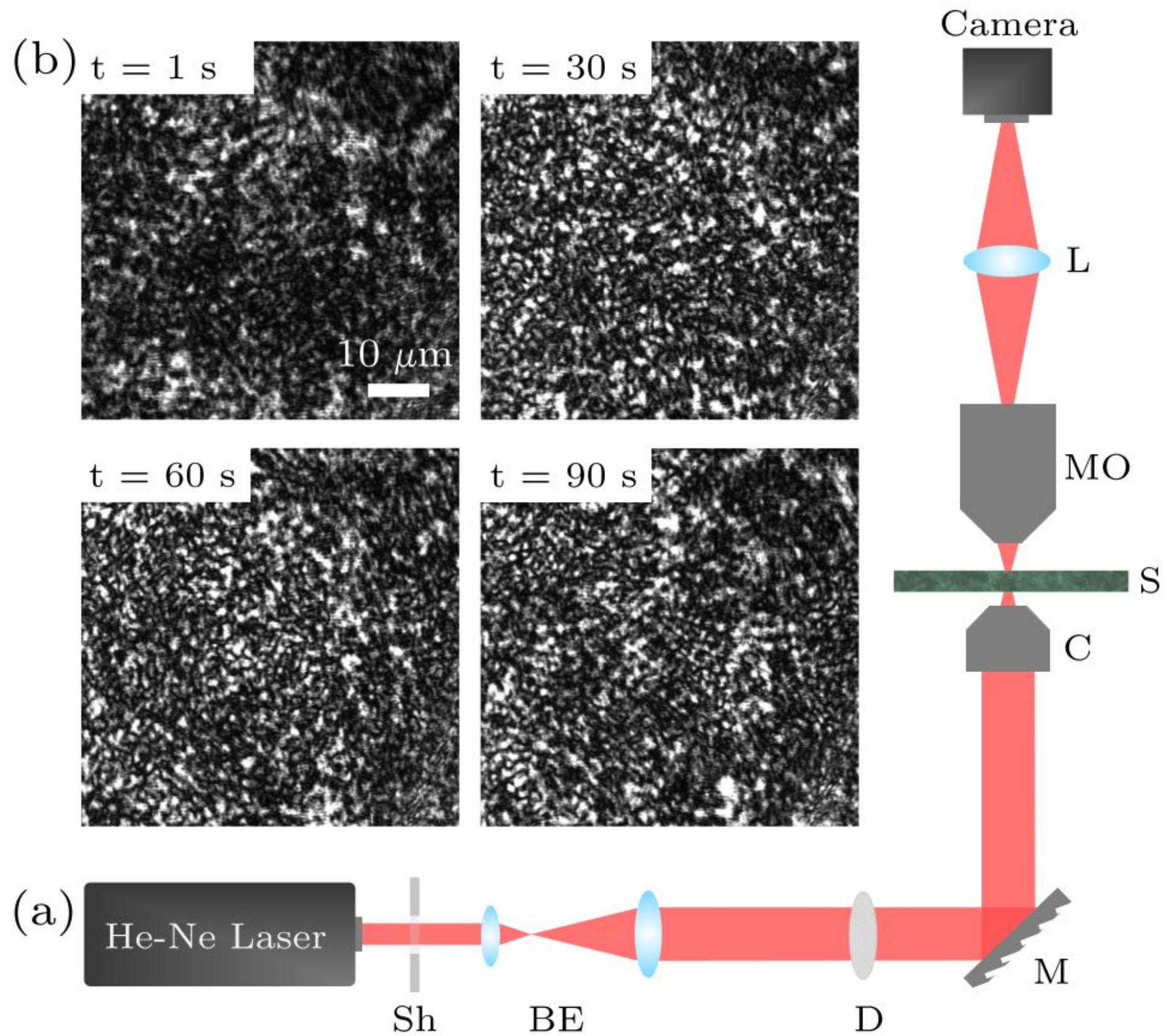

Figure 1

(a) Experimental setup schematically for dynamic speckle capture; Sh: shutter, BE: beam expander, D: Diffuser, M: mirror, C: Condenser, S: Sample, MO: Microscope Objective, L: collecting lens. (b) Speckle patterns of domain evolution over times. 
(a)



(b)

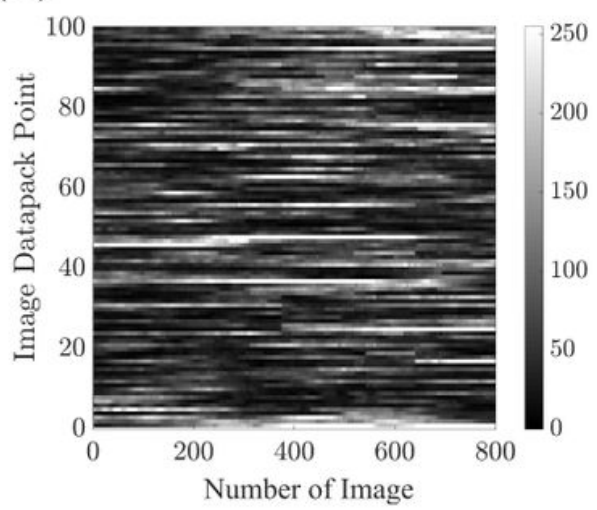

(c)

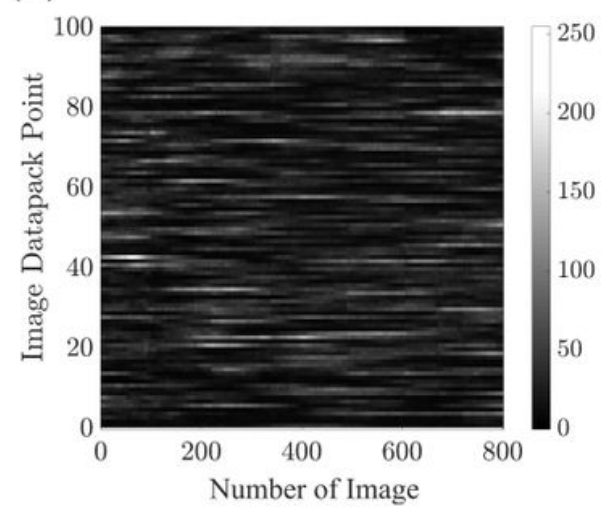

Figure 2

Time history speckle pattern (THSP) for (a) 10\%, (b) 20\%, and (c) 30\% Chol. THSP formed by tracking 100 random points throughout a collection of 810 speckle patterns of lipid (image datapack points). Speckle pattern recorded every second while domain evolution.

(a)

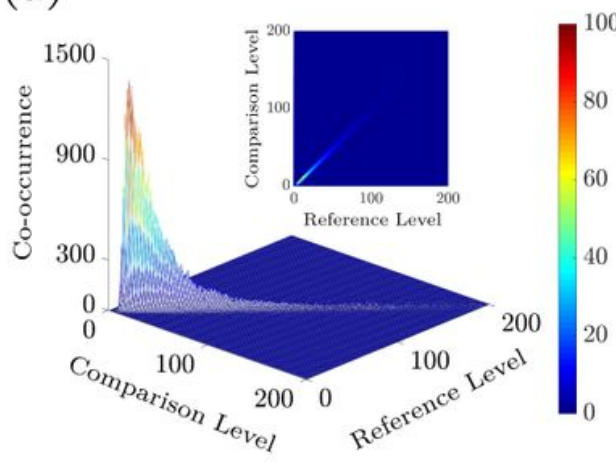

(b)

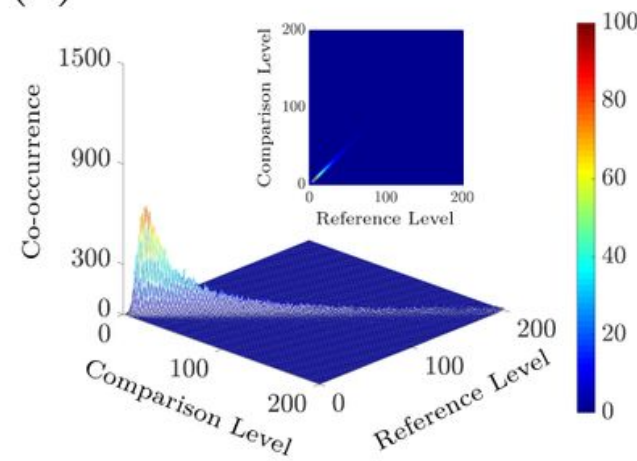

(c)

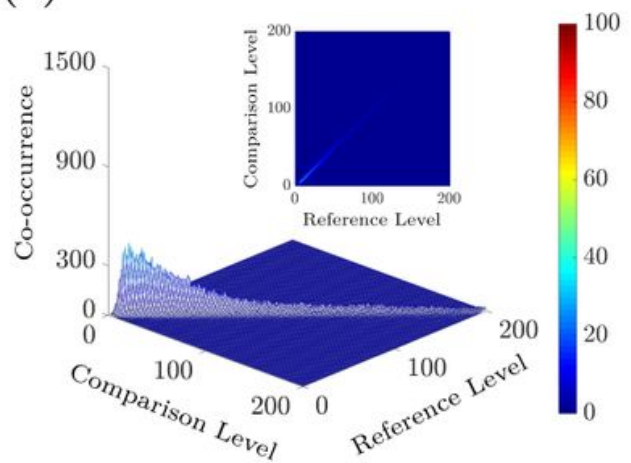

Figure 3

3D plot and 2D map of co-occurrence matrix (COM) of the associated THSPs in Fig. 2 for (a) 10\%, (b) $20 \%$, and (c) $30 \%$ Chol.

(a)

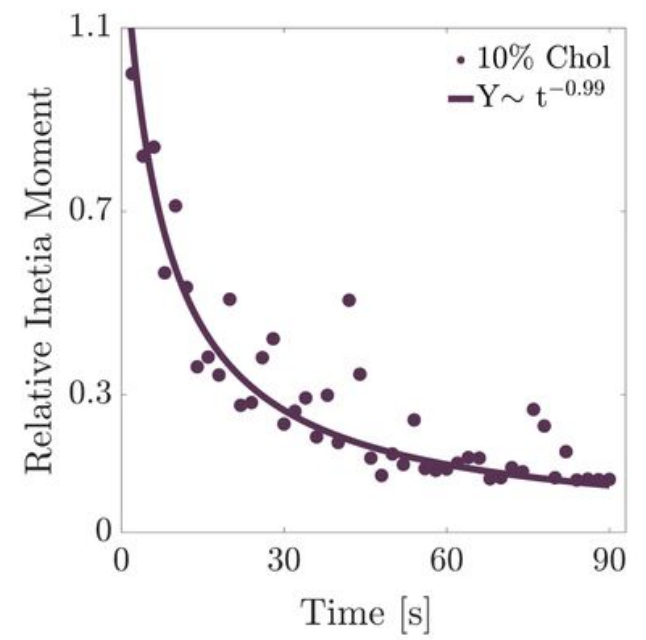

(b)

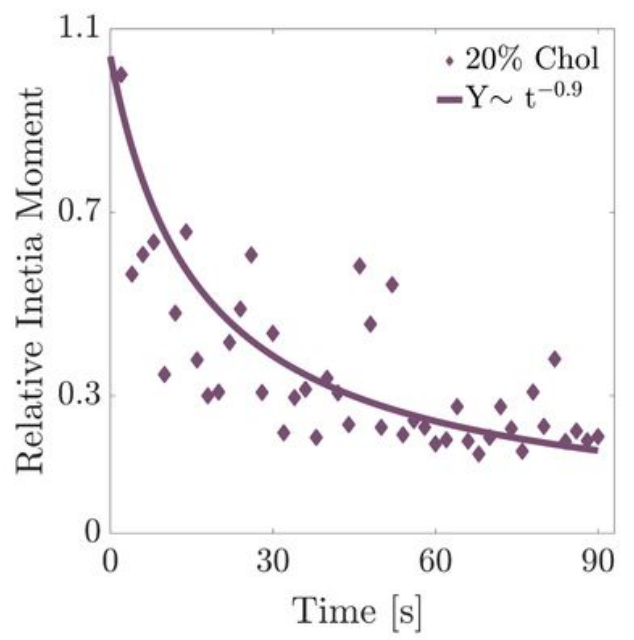

(c)

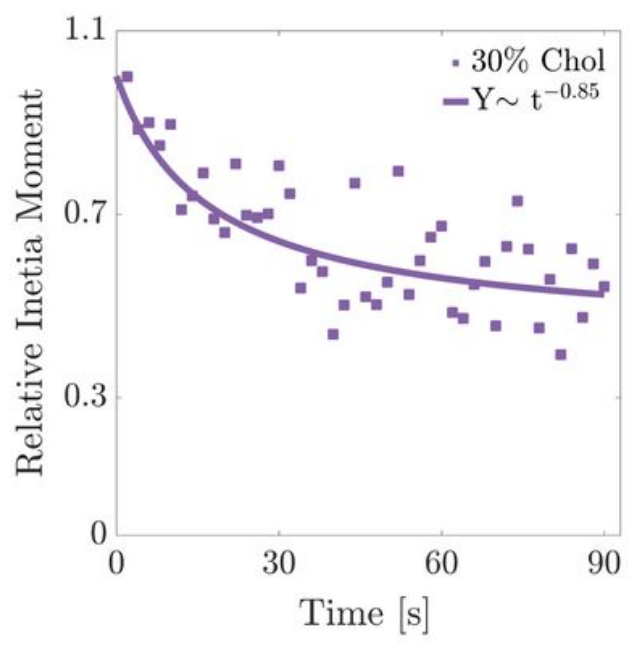


Figure 4

Inertia moment (IM) of lipid samples' THSP as a function of time for (a) $10 \%$, (b) $20 \%$, (c) $30 \%$ Chol. The power-law function is fitted on each graph.

(a)

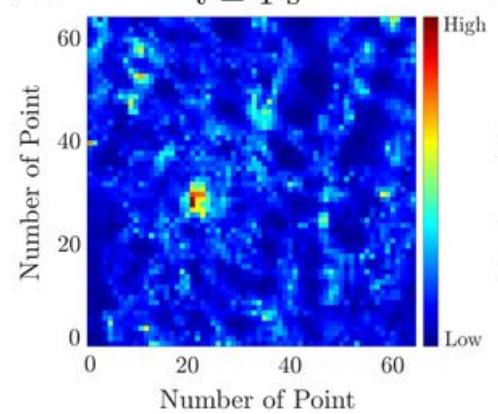

(b)

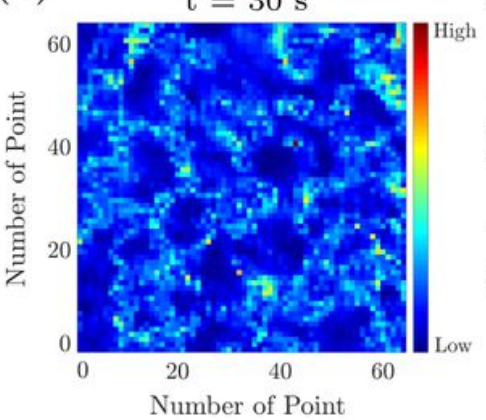

(f)

(e)


(c)

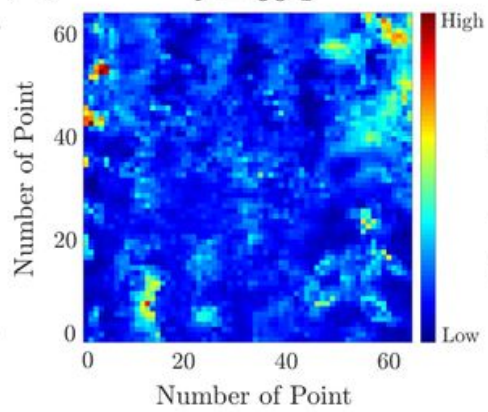

(d)

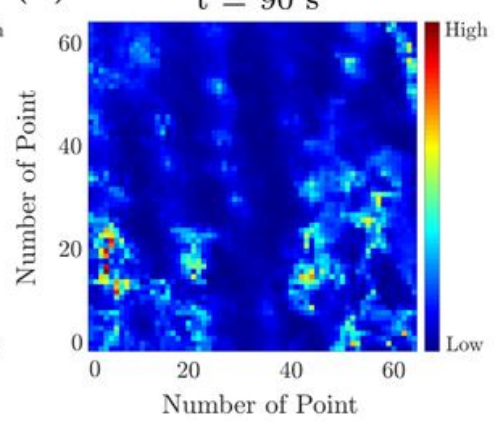

(h)

$\mathrm{t}=90 \mathrm{~s}$

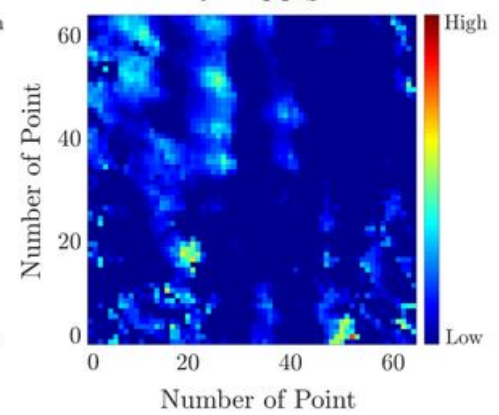

Number of Point

Figure 5

(a), (b), (c), and (d) Temporal speckle kurtosis matrix of $20 \%$ Chol. (e), (f), (g), and (h) Temporal speckle skewness matrix of $20 \%$ Chol.

(a)

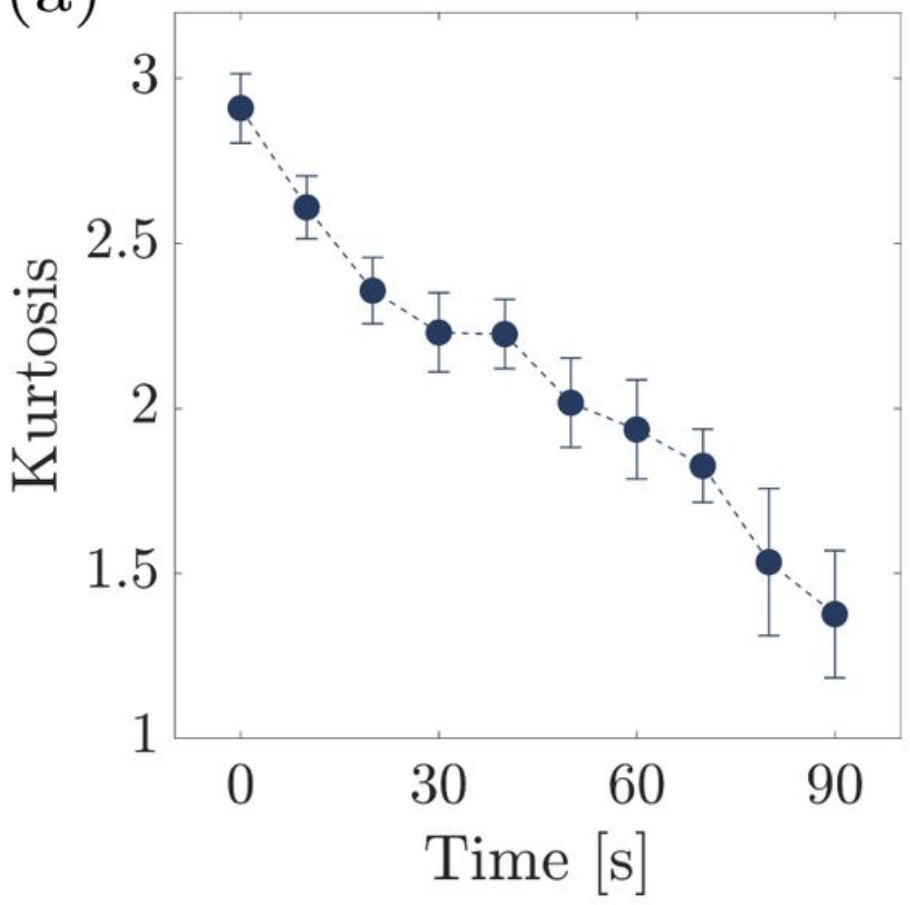

(b)

(g) $\quad \mathrm{t}=60 \mathrm{~s}$

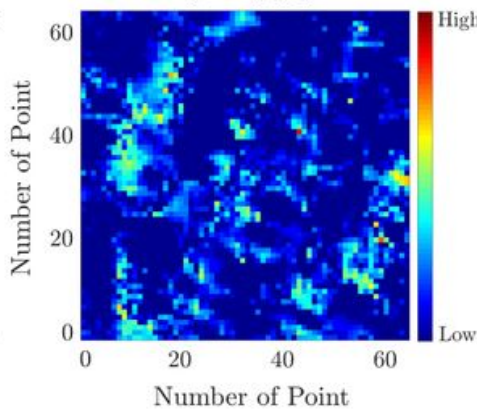


Figure 6

(a) Average kurtosis, and (b) Average skewness of 100 speckle patterns of lipid surface as a function of time at $20 \%$ Chol.
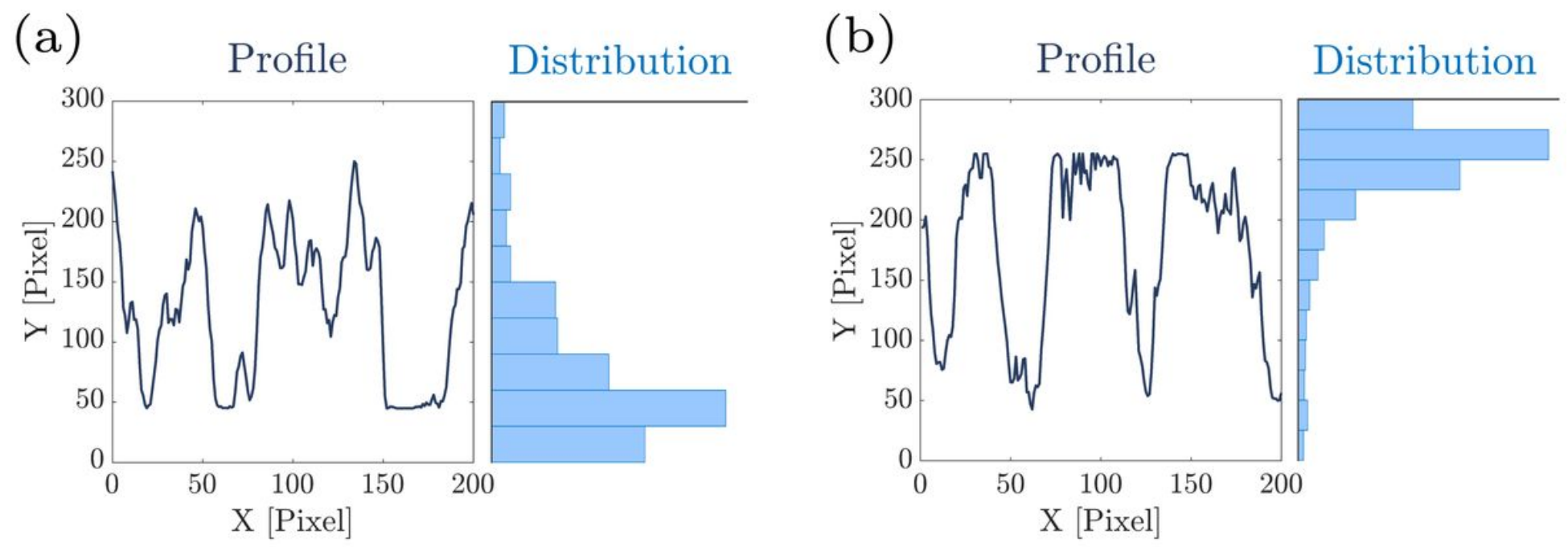

\section{Figure 7}

The intensity profile of two speckle patterns and corresponding intensity histograms at two different times, (a) $t=10 \mathrm{~s}$ and (b) $t=90 \mathrm{~s}$, illustrate positive and negative skewness respectively.

(a)

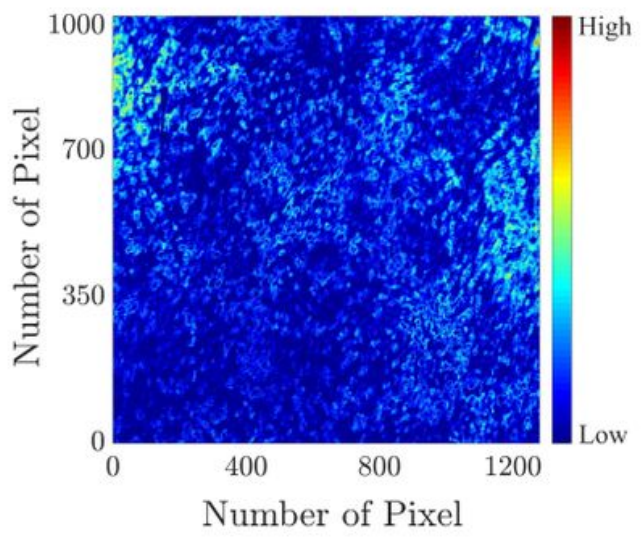

(b)

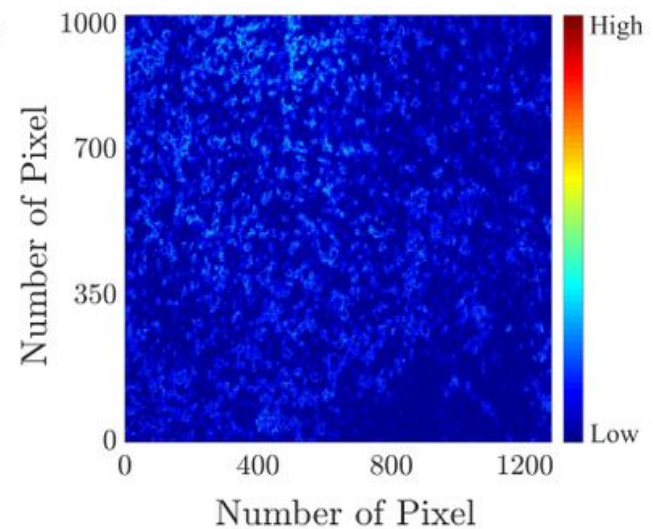

(c)

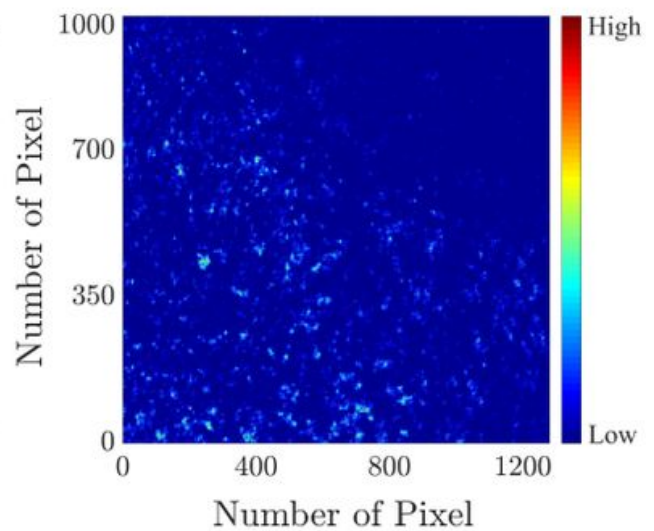

\section{Figure 8}

Motion history image (MHI) for (a) 10\%, (b) 20\%, (c) 30\% Chol. Each MHI was generated from a stack of speckle pattern images.

\section{Supplementary Files}

This is a list of supplementary files associated with this preprint. Click to download. 
- SR2020SpLipidSupplementaryMaterials.pdf 